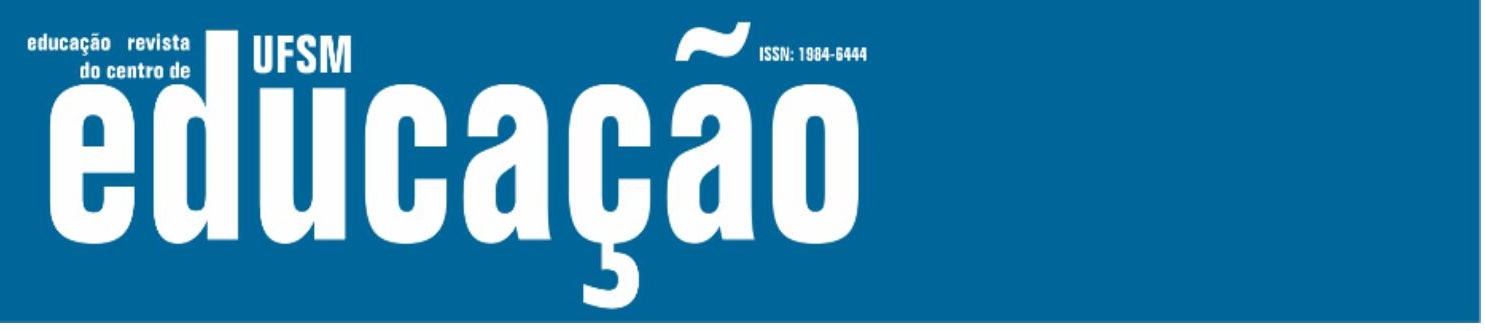

ISSN: 1984-6444 | http://dx.doi.org/10.5902/1984644443020

\title{
Formação professores e processo educativo: a Companhia de Jesus nos clássicos da educação brasileira
}

Teacher education and educational process: the Society of Jesus in the brazilian education's classics

\section{Patrícia Fontana}

Mestra em Educação pela Universidade Regional Integrada do Alto Uruguai e das Missões, Campus Frederico Westphalen, Rio Grande do Sul, Brasil.

patricia.fontanaimm@gmail.com - http://orcid.org/0000-0002-3843-1290

Martin Kuhn

Professor Doutor na Sociedade Educacional Três de Maio - SETREM - Três de Maio, Rio Grande do Sul, Brasil.

martk@outlook.com.br - http://orcid.org/0000-0002-8107-0814

Tatiane de Freitas Ermel

Pesquisadora pós-doutoral da Universidad Complutense de Madrid. Madrid, Espanha.

tatiane.ermel@gmail.com - https://orcid.org/0000-0003-2002-5101

Recebido em 19 de março de 2020

Aprovado em 22 de fevereiro de 2021

Publicado em 31 de julho de 2021

\section{RESUMO}

O presente artigo analisa três obras clássicas da educação brasileira, $A$ Cultura Brasileira: Introdução ao estudo da cultura no Brasil (1943), de autoria de Fernando de Azevedo; O método pedagógico dos jesuítas: o Ratio Studiorum (1952), de Leonel Franca e História da Educação Brasileira (1972), de José Antonio Tobias, fazendo um movimento em direção à tradição e refletindo acerca da formação de educadores e os processos educativos ao (re)visitar a tradição da Companhia de Jesus. Objetiva-se, desse modo, dialogar com a tradição para compreender o legado, mas fundamentalmente, o que podemos aprender dela sobre a formação de professores e o processo educativo. O estudo está organizado em dois movimentos: primeiro, a contextualização histórica do período jesuítico, em seguida, o aprofundamento do método pedagógico dos jesuítas e a formação de seus educadores, referida pelo Ratio Studiorum. Do ponto de vista teórico e metodológico, situa-se no âmbito da linguagem e da teoria de recepção e a recontextualização do conhecimento educativo.

Palavras-chave: Tradição; Pedagogia jesuítica; Formação de educadores. 


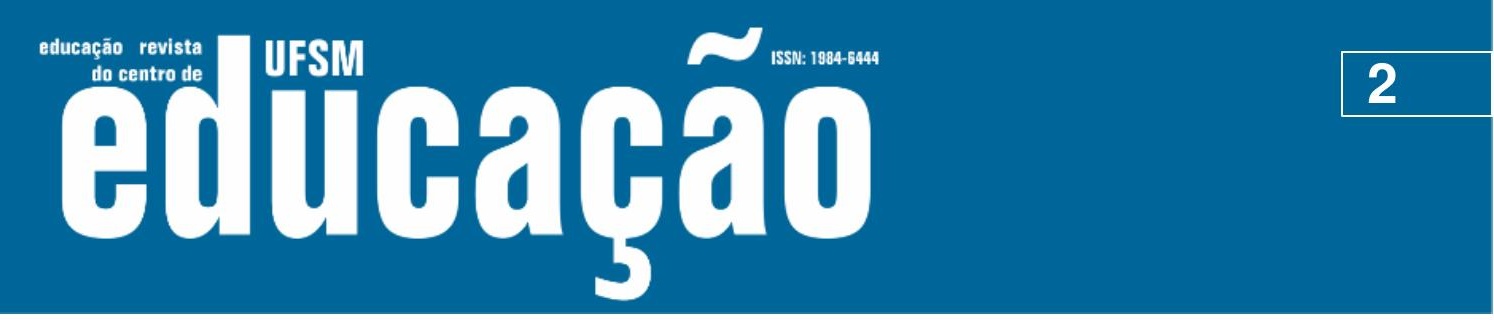

ISSN: 1984-6444 | http://dx.doi.org/10.5902/1984644443020

\section{ABSTRACT}

This paper analyzes three Brazilian education classics, A Cultura Brasileira: Introdução à Cultura no Brasil ("The Brazilian Culture: Introduction to the Study of Culture in Brazil"), from 1943, by Fernando de Azevedo; the Jesuit educational method, known as Ratio Studiorum (1952), by Leonel Franca; and História da Educação Brasileira ("History of Brazilian Education"), from 1972, by Jose Antonio Tobias, addressing tradition and reflecting on teacher education and on the educational processes in the context of (re)visiting the tradition of the Society of Jesus. Thus, the goal is to approach tradition to understand the legacy, and basically to identify what we can learn from it when discussing teacher education and educational processes. The study is organized in two movements: the first one regards the historical contextualization of the Jesuit period, in addition to the deepening of their pedagogical method and the education of their teachers, according to the Ratio Studiorum. The theoretical and methodological perspective is grounded on language and on the theory of reception and recontextualization of the educational knowledge.

Keywords: Tradition; Jesuit education; Teacher education.

\section{Introdução}

A construção deste artigo provém da seguinte inquietação: o que podemos aprender sobre a formação de educadores e sobre os processos educativos ao (re)visitar a tradição da Companhia de Jesus? Destacamos que a tradição sempre tem algo a nos ensinar. $O$ dialogar com a tradição nos permite conhecer $e$ reconhecer o que fizeram os que vieram antes de nós e que temos algo a aprender.

Para abordar a tradição da Companhia de Jesus recorremos a três autores clássicos ${ }^{1}$ da história da educação brasileira: José Antonio Tobias, Fernando de Azevedo e o Pe. Leonel Franca. Ao interpretar a tradição jesuítica de educação a partir destes autores clássicos, reconhecemos que o olhar de cada autor marca e insere o leitor em uma determinada forma de ver/compreender a tradição. Pois, ao valermo-nos somente de leituras de comentadores, interpretadores ou de leitores críticos corremos o risco de limitar à compreensão cultural presente em cada uma das obras. 


\section{Fism Ellthapao

ISSN: 1984-6444 | http://dx.doi.org/10.5902/1984644443020

"o primeiro século foi, pois, o de adaptação e construção, e o segundo, de desenvolvimento e extensão do sistema educacional que, adquirida a altura necessária foi alargando progressivamente, com unidades escolares novas, a sua esfera de ação". Assim, o século (XVI) foi, para os jesuítas, um tempo de adaptação, de investir na formação do ser humano, de catequizar e propagar a fé, enquanto o século seguinte (XVII) foi o desabrochar educacional.

A implementação de uma proposta educacional, seja em estruturas de escolas e qualificação de saberes, formação de educadores foi um processo que demandou paciência, empenho e qualificação. De acordo com Teive (2007, p.60), os colégios coloniais formaram uma base administrativa dos jesuítas e concentraram diferentes ações, tais como: "alfabetização dos indígenas, ensino de artes e ofícios para indígenas e escravos, ensino de gramática latina para filhos de colonizadores e pretendentes do clero, cultivo de hortas, pomares e criação de animais, entre outras". Destaca ainda que estes colégios se tornaram espaços de referência da "sociabilidade da civilização cristã ocidental", através das bibliotecas, enfermarias, oficinas e boticas que prestaram assistência à população em geral.

Conforme Tobias (1986, p. 72), foi depois de um século que as sementes lançadas pelos jesuítas começam a desabrochar por meio de novas unidades escolares e também pelo surgimento de outras áreas de ensino. Salienta que "Nóbrega foi o personagem da primeira educação brasileira, que se estende de 1545 a 1759, ano da expulsão dos jesuítas. Provincial, líder, homem de visão, estadista e emérito educador". Nóbrega era uma pessoa com vasto conhecimento, identificado com $o$ ato de ensinar e com liderança fez a diferença no nascente sistema educacional brasileiro, sendo que ele "ministrou a educação realmente democrática, abrasileiradora e realista, cristã e humana, um modelo de educação, digna do Brasil e de ser continuada e ampliada" (Idem, p. 72). Após os desafios do primeiro século, a educação jesuítica se consolida e constrói sua identidade com a elaboração o método pedagógico próprio (Ratio Studiorum) e será reconhecida como um dos principais correntes de educação e cultura da época moderna. 


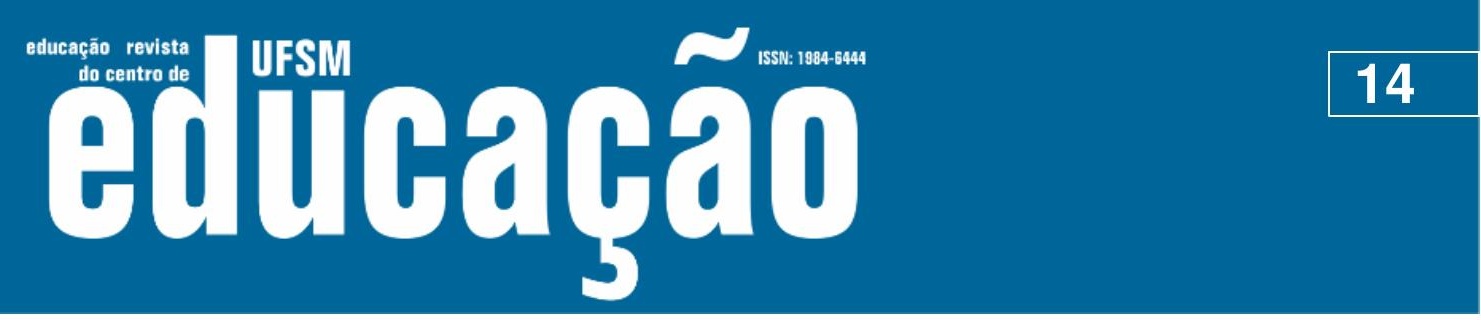

ISSN: 1984-6444 | http://dx.doi.org/10.5902/1984644443020

Franca (1952, p. 46), destaca também que o "Braço direito do reitor, na orientação pedagógica, é o prefeito de estudos. Homem de doutrina e de larga experiência no ensino [...]". As características exigidas do prefeito de estudos são relevantes para o sucesso da educação jesuítica. De acordo com o autor (1952, p. 46), o prefeito de estudos acompanha a vida escolar "visita periodicamente as aulas, urge a execução dos programas e dos regulamentos, forma e aconselha os novos professores, articula a atividade de todos. Guarda fiel das tradições, assegura, com a unidade atual da obra pedagógica, a sua continuidade no tempo". A função delegada ao prefeito de estudos revela a seriedade no acompanhamento dos docentes no desenvolvimento da tarefa educativa. Assim, se havia exigências, também eram oferecidas possibilidades de formação continuada e ajuda por parte dos prefeitos de estudos, que eram preparados para acompanhar a vida escolar e os professores, pois há clareza de que o êxito do processo educativo dependeria de professores identificados e qualificados no método.

Diversos eram os saberes docentes que constituíam a identidade do professor e que eram mobilizados na organização e execução das aulas. Franca (1952, p. 50) ressalta que "Para julgar com acerto este currículo (Teológico, Filosófico, Humanista) e a ausência de disciplinas que hoje nos parecem indispensáveis num curso secundário, convém lembrar a situação cultural do século XVI". Pondera que as ciências experimentais não "haviam tomado o desenvolvimento que hoje conhecemos nem as línguas modernas a importância que Ihes deu posteriormente o surto progressivo das nacionalidades e o enriquecimento das respectivas literaturas". O currículo proposto pelo Ratio ainda estava distante das ciências experimentais e que foi uma das razões alegadas por Pombal para justificar a expulsão da Companhia de Jesus do Reino e de suas colônias, em 1759. Neste sentido, reforça-se a compreensão de que a formação do professor e o desenvolvimento do processo educativo são produções históricas e o método jesuítico é expressão de seu tempo.

Os jesuítas foram flexíveis também na organização do currículo ao longo da história e na ampliação de disciplinas e saberes que constituíam requisito para ser professor. Franca (1952, p. 54) destaca que "com o correr dos anos e o 


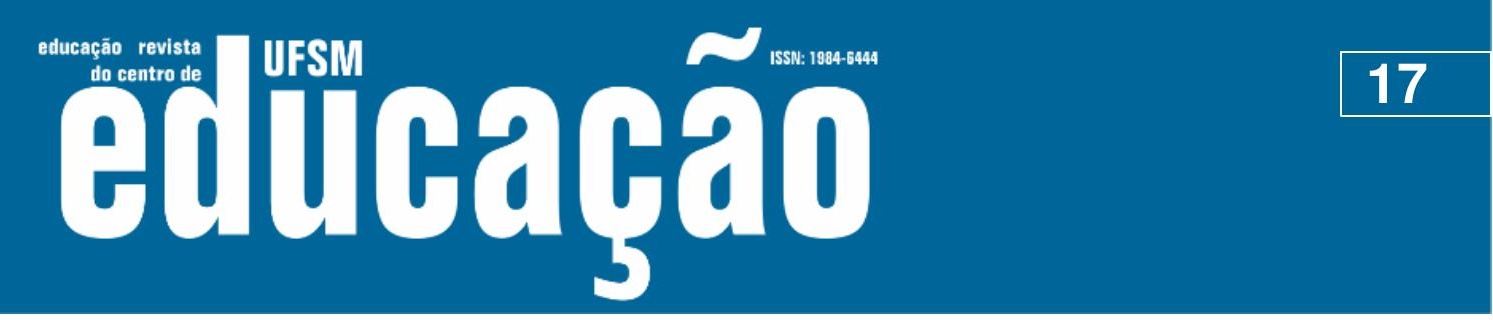

ISSN: 1984-6444 | http://dx.doi.org/10.5902/1984644443020

\section{Entre passado (s), presente (s) e futuro (s) da formação de professores e dos processos educativos}

Destacamos, como ponto de partida, que a tradição possibilita alargar o entendimento do contexto em que estamos inseridos. Para Marques (1990, p. 94), o entrar na tradição proporciona reconhecer "que o passado e o presente se acham em contínua mediação de reciprocidades, leva a uma compreensão inteligente das situações mudadas, em que o importante não é o que aconteceu, mas o que não cessa de acontecer". Pondera que "compreender é entrar no acontecimento da tradição, é produzir a significação efetivadora e atualizadora da consciência histórica". Neste sentido, Kuhn e Toso (2016, p. 93) apontam que quando esclarecida a tradição acumulada essa "pode ajudar a interpretar as novas questões e desafios que a realidade apresenta. Escavar o sedimento constitui possibilidade de reabertura, que se funda na tradição, para pensar sempre de modo novo a tarefa educativa do presente". Esse movimento abre possibilidades para (re) pensar, (re) criar e (re) significar tanto o processo de formação docente como o processo educativo.

Acerca da compreensão da partícula "re" que aparece reiteradamente, Fontana (2000, p. 157) levanta um questionamento: "o que significa esse (re) que a gente tanto usa"? Ele remete a ideia de voltar a alguma coisa, fazer de novo: "voltar a fazer, voltar a planejar, ver de novo, fazer de novo, planejar de novo". É relevante este movimento de inacabamento de idas e vindas quando se trata de itinerários formativos. Por isso, conhecer a tradição, voltar a ela nos permite fazer de outro modo. Aprender com o vivido, mas de um modo novo. Compreendemos que é no "diálogo com a tradição, de nossas deliberações acerca do que queremos da educação, de nossas opções emancipatórias, que se constitui a identidade da escola e do professor" (KUHN, 2016 p. 211). Assim o retorno à história não é distanciar-se do contexto presente, mas buscar compreendê-lo de forma mais profunda e é este movimento que possibilita "pensar de modo novo". Interpretar e compreender a tradição abre a possibilidade de o sujeito docente reconhecer as marcas da historicidade presente na constituição de sua identidade. 


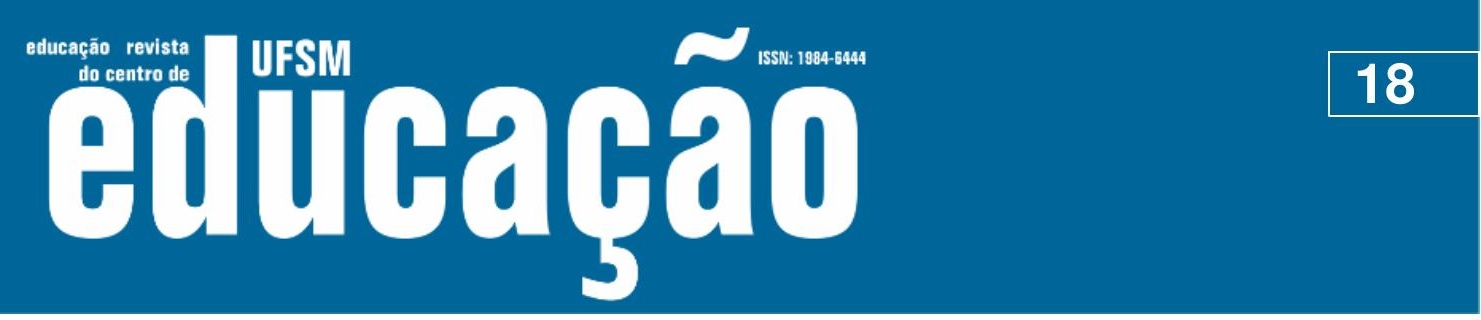

ISSN: 1984-6444 | http://dx.doi.org/10.5902/1984644443020

O que podemos aprender sobre a formação de educadores e sobre os processos educativos ao (re)visitar a tradição da Companhia de Jesus? Se há muitas críticas dirigidas à tradição jesuítica, e não sem razão, contudo, não significa que nada há a aprender com ela. Os dois aspectos centrais dessa tradição têm algo a nos ensinar: o primeiro diz do compromisso assumido pelo Ratio Studiorum com a formação do educador jesuíta e o segundo diz da pré-ocupação com o processo instrutivo/educativo.

Em relação ao primeiro aspecto, observamos o compromisso da Ordem com a formação do educador jesuíta e destacamos a sólida formação em termos de conhecimentos requeridos para o exercício da docência. Ainda em relação à formação do professor, merece atenção o cuidado da congregação com o professor iniciante. O acompanhamento de um "docendi peritissimum" é outro aspecto que merece destaque. Assim, o aspirante a professor, ao concluir sua formação e assumir propriamente à docência, era acompanhado por um professor experiente, versado nas práticas do magistério, que o iniciava no ofício de bom professor.

Assim, a tradição jesuítica nos permite questionar pelo menos dois pontos em nosso atual processo de formação de educadores: a) a solidez dos conhecimentos gerais, dos conhecimentos pedagógicos e dos conhecimentos específicos da formação do educador e b) a iniciação à docência. Hoje, os currículos dos cursos de licenciatura encontram-se cada vez mais esvaziados de conhecimentos teóricos, pedagógicos e disciplinares e são largamente reduzidos a uma razão instrumental, cada vez mais operacional. Estágios ao longo da formação, ao final da formação, PIBID, Residência Pedagógica, etc., todas são estratégias de aproximação do futuro docente das práticas educativas. Contudo, parece-nos que o cuidado da pedagogia jesuítica com o iniciante à docência, acompanhado por um sujeito versado na vida escolar, nos programas escolares, nos regulamentos, que forma e aconselha os novos professores, extrapola em muito os nossos reducionismos.

Assim, essa dupla exigência da formação dos educadores jesuítas questiona os nossos cursos de licenciatura, pois usualmente temos algumas horas de estágio, durante e antes de concluir o curso e uma vez 'formado' o docente iniciante chega à escola, sem muita experiência pedagógica e, em muitas ocasiões, sem o devido 


\section{Tusm

ISSN: 1984-6444 | http://dx.doi.org/10.5902/1984644443020

de seu conhecimento. A pedagogia brasílica e a ação proposta por Anchieta junto aos indígenas é um exemplo inspirador da flexibilidade do método. Apesar de seu caráter metafísico, a pedagogia jesuítica acredita no potencial do ser humano (livre arbítrio), reconhece-o como alguém dotado de inteligência que precisa ser instigado a desenvolvê-la, isso parece atual.

O movimento de retornar à tradição para compreendê-la, não se trata de fazer apologia a ela, contudo, possibilita tomar pre-sença memórias que contribuem para enfrentarmos os desafios que se apresentam à educação, a formação de professores, às práticas educativas de nosso tempo. Neste sentido, é válido relembrar a frase dita na introdução, a tradição sempre tem algo a nos ensinar. Assim, algumas exigências postas em nosso tempo para a docência, para a iniciação à docência, mesmo orientações metodológicas, parecem não ser tão novas quanto imaginamos. O retornar à tradição permite compreender as heranças deixadas pelos que vieram antes. O método pedagógico jesuítico previa exigências consideradas importantes para o seu tempo, mas que deixaram marcas indeléveis em nossa tradição educativa e podem ajudar a pensar os desafios do nosso tempo.

\section{Referências}

ARANHA, Maria Lúcia de Arruda. História da Educação e da Pedagogia, Geral e Brasil. São Paulo: Moderna, 2006.

AZEVEDO, Fernando de. A Cultura Brasileira: Introdução ao estudo da cultura no Brasil. Brasília: Universidade de Brasília, 1963.

BASTOS, Maria Helena Camara. Maria Lúcia Aranha e a História da Educação. Cadernos de História da Educação, Uberlândia/MG, v. 13, n. 2, p.517-534, jul./dez. 2014. (23/02/2015).

Disponível em:http://www.seer.ufu.br/index.php/che/article/view/29201. Acesso em: 15 nov. 2019.

BURKE, Peter. The history and theory of reception. In: LLOYD, Howell A. (ed). Reception of Bodin. Brill: Leiden, Boston, 2013, p. 21-38.

CALVINO, Ítalo. Porque Ler os Clássicos. São Paulo: Companhia das Letras, 1993. 


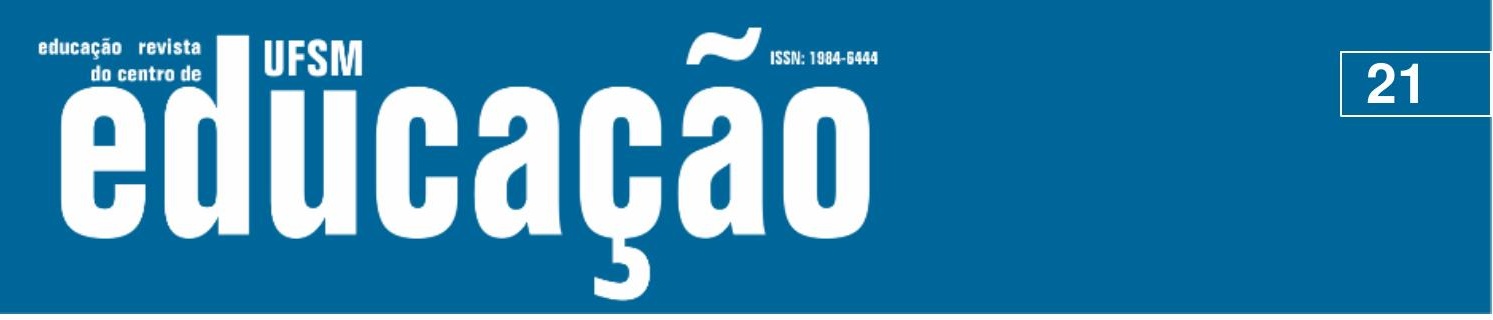

ISSN: 1984-6444 | http://dx.doi.org/10.5902/1984644443020

FONTANA, Roseli Canção. Como nos Tornamos Professoras? Belo Horizonte: Autêntica, 2000.

FRANCA, Leonel. O método pedagógico dos jesuítas: o Ratio Studiorum. Rio de Janeiro: Agir, 1952.

HEIDEGGER, Martin. Ser e tempo. Petrópolis/RJ: Editora Vozes, 2002.

KOSELLECK, Reinhart. Estratos do tempo: estudos sobre história. 1. ed. Rio de Janeiro: Contraponto: PUC-Rio, 2014.

KUHN, Martin. O professor: identidade e protagonismo. 2016. 261 p. Tese (Doutorado em Educação nas Ciências), Universidade Regional do Noroeste do Estado do Rio Grande do Sul, ljuí 2016.

KUHN, Martin; TOSO, Cláudia Eliane Ilgenfritz. Epistemologias na Formação Docente. In: ANDRADE, Elisabete (org.). Políticas Educacionais e Formação de Professores. Curitiba: CRV, 2016. p. 83 - 97.

LEITE, Serafim. Artes e Ofícios dos Jesuítas no Brasil (1549-1760). Lisboa: Editora Brotéria, 1953.

MARQUES, Osorio Mario. Pedagogia a Ciência do Educador. Ijuí/RS: Unijuí, 1990.

MESNARD, Pierre. La pedagogía de los jesuitas. In: CHÂTEAU, Jean (Org.). Los grandes pedagogos. México: Fundo de Cultura Económica, 2017, p. 59-126.

PAIVA, José Maria de. Colonização e catequese. São Paulo: Autores Associados: Cortez Editora, 1981.

SCHRIEWER, Jürgen. Deweyan Thought Refracted Through Time and Space. Studies on the Trans-Continental Dissemination and Culture-Specific ReContextualization of Educational Knoweledge. In: BRUNO-JOFRÉ, Rosa (Org.). The Global Reception of John Dewey's Thought: Multiple refractions through time and space. New York \& London: Routledge - forthcoming, 2012, p.1-19.

TOBIAS, José Antônio. História da Educação Brasileira. São Paulo: Ibrasa, 1986.

TEIVE, Cyntia Greive. História da Educação. São Paulo: Ática, 2007.

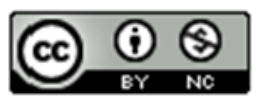

This work is licensed under a Creative Commons Attribution-NonCommercial 4.0 International (CC BY-NC 4.0) 


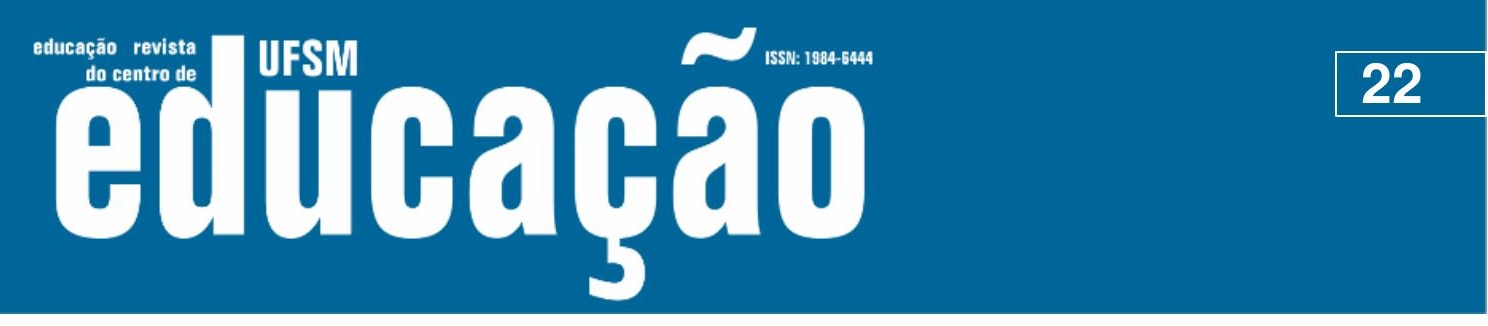

ISSN: 1984-6444 | http://dx.doi.org/10.5902/1984644443020

\title{
Notas
}

\begin{abstract}
1 De acordo com Calvino (1993, p. 11) "os clássicos são aqueles livros que chegam até nós trazendo consigo as marcas das leituras que precederam a nossa e atrás de si os traços que deixaram na cultura ou nas culturas que atravessaram (ou mais simplesmente na linguagem ou nos costumes)". Destaca que "nunca será demais recomendar a leitura direta dos textos originais, evitando o mais possível bibliografia crítica, comentários e interpretações" (1993, p. 12).
\end{abstract}

2 Entre parênteses indicamos as datas das primeiras edições de cada obra, mas para o presente estudo utilizamos edições de 1963, de Fernando de Azevedo e, de 1986 de José Antonio Tobias.

3 "Inácio de Loyola (1491-1556), militar espanhol basco, ao se recuperar de um ferimento em batalha, viu-se envolvido por súbito ardor religioso e resolveu colocar-se a serviço da defesa da fé, tornandose verdadeiro 'soldado de Cristo'. Fundou então a Companhia de Jesus, daí o nome jesuítas dado aos seus seguidores" (ARANHA, 2006, p. 127).

${ }^{4}$ Suas bases foram "lançadas em 15 de agosto de 1534 na capela Montmartre por Inácio de Loyola e seus seis companheiros" (AZEVEDO,1963, p. 501).

${ }^{5}$ Para um estudo aprofundado sobre a pedagogia jesuíta, sua organização e expansão na Europa, ver Mesnard (2017).

${ }^{6}$ De acordo com Teive (2007, p. 55), a Companhia de Jesus ao chegar no Brasil, em 1549, com a esquadra que trouxe o primeiro Governador Geral, Tomé de Souza, veio acompanhada por 5 ou 6 padres, que acompanhavam Manuel de Nóbrega, seu superior. Quando da expulsão da Ordem, em 1759, havia aproximadamente 510 jesuítas no Brasil, excluindo os que viviam nos aldeamentos. Para mais detalhes ver Serafim Leite (1953).

7 Médio é a nomenclatura utilizada por Tobias (1986), já Franca (1952, p. 47) usa a expressão secundário, termo que está no Ratio Studiorum.

8 Padre Cláudio Aquaviva responsável de coordenar o trabalho de elaboração do Ratio Studiorum.

9 Em 1550 a congregação funda a Escola Normal com a tarefa de preparar "entre os estudantes da Ordem, os futuros professores, adestrando-os nos melhores métodos e pondo-os em contacto imediato com os educadores mais abalizados" (FRANCA, 1952, p. 10).

10 Contudo, para além da qualidade da formação humana, da instrução e da sólida formação dos educadores, o Plano de Estudos sugere que o educador "[...] consagre aos alunos um afeto paterno, mas sem familiaridades; trate a todos com bondade e justiça, não despreze a ninguém, nem faça distinção entre rico e pobre; não seja precipitado em castigar nem demasiado em inquirir; dissimule muitos defeitos; não só não bata no aluno, mas nem sequer lhe dirija palavra injuriosa, ou o chame senão pelo seu nome ou cognome" (FRANCA, 1952, p. 90).

${ }^{1} 1$ Sob o nome metodologia a Ratio Studiorum compreendia "tanto os processos didáticos adotados para a transmissão de conhecimentos, quanto os estímulos pedagógicos postos em ação para assegurar o êxito do esforço educativo" (FRANCA, 1952, p. 56),

12 No curso de formação humanista, "O aluno deve desenvolver todas as suas faculdades [...]. As classes de gramática asseguram-Ihes expressão clara e exata, a de humanidades, uma expressão rica e elegante, a da retórica mestria perfeita na expressão poderosa e convincente ad perfectuam eloquentiam informat' (FRANCA, 1952, p. 49). 